\title{
QSAR, Docking and invitro antioxidant activity study of novel chromone derivatives
}

\author{
Shanthi V. ${ }^{1, *}$, Ramesh M., Srimai V., Srinivas P. ${ }^{3}$, Parthasarathy T. ${ }^{2}$ \\ ${ }^{1}$ Muffakhamjah College of Engineering and Technology Hyderabad \\ ${ }^{2}$ Department of Chemistry, University College Of Science, Saifabad, Osmania University, Hyderabad, Andhra Pradesh, India \\ ${ }^{3}$ Department of Chemistry, University College Of Science, Osmania University, Hyderabad, Andhra Pradesh, India
}

\section{Email address:}

sarathychem@gmail.com (Shanthi V.)

\section{To cite this article:}

Shanthi V., Ramesh M., Srimai V., Srinivas P., Parthasarathy T.. QSAR, Docking and Invitro Antioxidant Activity Study of Novel Chromone Derivatives, Modern Chemistry. Vol. 1, No. 1, 2013, pp. 8-17. doi: 10.11648/j.mc.20130101.12

\begin{abstract}
Invitro antioxidant activity and Molecular modeling studies of nine chromone derivatives were carried out to optimize their inhibitory activity against Poly [ADP-ribose] polymerase. The biological activities of these analogs were correlated to different molecular properties. The AM1 and PM3 semiempirical methods are used to estimate vertical ionization potentials (IPv's), electron affinity (EA), electronegativity $(\chi)$, hardness $(\eta)$, softness (S), electrophilic index $(\omega)$, partition coefficient (LogP), hydration energy (HE), ionization potential (IP) and charges. The different modeled equations by regression analysis are proposed. The leave-one-out cross-validation method is used to estimate the predictive power of final QSAR equations. The hardness $(\eta)$ was found to be indicative molecular property by regression analysis. Docking studies of chromone with Poly [ADP-ribose] polymerase are also made to support the finding of QSAR studies. Analysis of results of both QSAR and Docking studies suggested that remarkable inhibitory activity is exhibited by molecule 3 . The hydrogen bond interactions along with hydrophobic and electrostatic interactions are mapped to confirm their potencies.
\end{abstract}

Keywords: Chromone Derivatives, Semi Empirical Methods, Cross-Validation Method, Inhibitor, QSAR, Regression Analysis, Docking

\section{Introduction}

Chromones (Figure 1) constitute one of the major classes of naturally occurring compounds, and interest in their chemistry continues unabated because of their usefulness as biologically active agents [1]. Chromone (or 1, 4benzopyrone) is a derivative of benzopyran with a substituted keto group on the pyran ring.The derivatives of chromone are collectively known as chromones.Chromones, i.e. 4H-1-benzopyran-4-ones, are very common motifs in natural products as well as drugs.

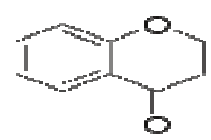

Figure 1. Chromone Nucleus.

Chromone derivatives are abundant in nature and possess a wide range of biological and pharmacological activity [2]. Some of the biological activities of chromone derivatives include cytotoxic (anticancer) [3,4,5], neuroprotective [6] ,
HIV-inhibitory[7], antimicrobial [8, 9], antifungal [10] and antioxidant activity[11]. Due to their abundance in plants and their low mammalian toxicity, chromone derivatives are present in large amounts in the diet of humans [12].

The synthesis of chromone derivatives is a research field of great interest and long history [13]. In general, chromones are synthesized by the cyclodehydration of 1- $(o-$ hydroxyaryl)-substances that favor healing of wounds[14] and ulcers [15] immunostimulators [16] and as anti-HIV agents [17]. Many chromone derivatives are also photoactive and can be used easily in various photo induced reactions affording diverse heterocyclic compounds [18].

Oxidative stress conditions - disruption of homeostatic balance between free radical generation and the production of naturally occurring antioxidants (e.g. glutathione and regulatory enzymes such as super oxide dismutase, catalase,and peroxidases) are recognized to be directly linked to the damage in numerous cell targets which can finally result in severe diseases namely liver toxicity, cardiovascular, and neuro generative disorders and cancer. The phenolic constituents are the largest group and comprise phenolic 
acids, anthocyanins, tannins,chromones etc they possess health promoting properties due to their activity as chain breaking antioxidants by radical scavenging or reduction of free radical formation[19].

The antioxidant capacity of chromones depends on their chemical structure. The position of hydroxyl groups and other features in the chemical structure are important for their antioxidant and free radical scavenging activities.

Reactive oxygen species and other reactive species induce oxidative DNA damage and consequent activation of the nuclear enzyme poly (ADP-ribose) polymerase 1 (PARP-1) (the most abundant isoform of the PARP enzyme family). PARP over activation depletes its substrate $\mathrm{NAD}^{+}$, slowing the rate of glycolysis, electron transport, and ATP formation, eventually leading to functional impairment or death of cells, as well as up-regulation of various proinflammatory pathways. Therefore, novel antioxidants and PARP inhibitors have entered clinical development for the experimental therapy of various cardiovascular and other diseases [20].

Poly (ADP-ribose) polymerase-1 (PARP-1) is a member of the PARP enzyme family consisting of PARP-1 and several recently identified novel poly (ADP-ribosylating) enzymes. PARP-1 is an abundant nuclear protein functioning as a DNA nick-sensor enzyme. Upon binding to DNA breaks, activated PARP cleaves $\mathrm{NAD}^{+}$into nicotinamide and ADP-ribose and polymerizes the latter onto nuclear acceptor proteins including histones, transcription factors, and PARP itself. Poly (ADP-ribosylation) contributes to DNA repair and to the maintenance of genomic stability. On the other hand, oxidative stress-induced over activation of PARP consumes NAD ${ }^{+}$and consequently ATP, culminating in cell dysfunction or necrosis. This cellular suicide mechanism has been implicated in the pathomechanism of stroke, myocardial ischemia, diabetes, diabetes-associated cardiovascular dysfunction, shock, traumatic central nervous system injury, arthritis, colitis, allergic encephalomyelitis, and various other forms of inflammation. The PARP has also been shown to associate with and regulate the function of several transcription factors. The special interest is the enhancement by PARP of nuclear factor $\kappa \mathrm{B}$-mediated transcription, which plays a central role in the expression of inflammatory cytokines, chemokines, adhesion molecules, and inflammatory mediators.

As chromones and their structural analogs are known to play an important protective role against Poly (ADP-ribose) polymerase [19]. So, there is a substitutional requirement of synthetic chromone derivatives to improve them as antioxidants and also as Poly (ADP-ribose) polymerase inhibitors. This prompted us (i) to gain insight into the structural features related to the antioxidant activity of the polyphenolic compounds and (ii) to suggest new substituents or structures with potentially enhanced antioxidants as Poly (ADP-ribose) polymerase inhibitors.

Hence, the antioxidant activity and inhibitory activity against Poly (ADP-ribose) polymerase of chromones has motivated great interest in correlating their biological activities with that of structural activities using QSAR and molecular modeling studies. The information rendered by QSAR models and the docking interactions may afford valuable clues to optimize the lead and design new potential inhibitors.

Table 2. Values obtained for the $A M_{1}$ computational method.

\begin{tabular}{|c|c|c|c|c|c|c|c|c|c|c|}
\hline Comp & ipv & Ip & EA & $\chi$ & $\eta$ & $\mathbf{S}$ & $\omega$ & $\log P$ & HE & Pol \\
\hline 1 & -9.1259 & -1.3644 & -9.6368 & 5.5006 & 4.1362 & .1209 & 3.6575 & 1.0200 & -2.8100 & 39.2100 \\
\hline 2 & -9.1397 & -1.3487 & -9.6437 & 5.4962 & 4.1475 & .1206 & 3.6418 & 1.0200 & -2.8100 & 39.2100 \\
\hline 3 & -9.2708 & -1.3710 & -9.7858 & 5.5784 & 4.2074 & .1188 & 3.6981 & 1.1900 & -2.4600 & 42.9700 \\
\hline 4 & -9.1618 & -1.2413 & -9.6143 & 5.4278 & 4.1865 & .1194 & 3.5185 & 2.6000 & -4.1600 & 52.5400 \\
\hline 5 & -9.1482 & -1.3281 & -9.5682 & 5.4482 & 4.1200 & .1214 & 3.6022 & 1.2400 & -3.9600 & 50.4600 \\
\hline 6 & -9.2836 & -1.3750 & -9.8077 & 5.5913 & 4.2164 & .1186 & 3.7073 & .8000 & -2.4400 & 41.1400 \\
\hline 7 & -9.2081 & -1.3423 & -9.6078 & 5.4751 & 4.1327 & .1210 & 3.6267 & .9700 & -2.8600 & 49.8200 \\
\hline 8 & -9.1507 & -1.3045 & -9.6219 & 5.4632 & 4.1587 & .1202 & 3.5884 & -.1900 & -2.9300 & 42.4000 \\
\hline 9 & -8.7566 & -1.5179 & -10.6057 & 6.0618 & 4.5439 & .1100 & 4.0433 & -.0600 & -7.2900 & 30.4100 \\
\hline
\end{tabular}


Table 3. values obtained for the PM3 computational method.

\begin{tabular}{|c|c|c|c|c|c|c|c|c|c|c|}
\hline Comp & ipv & ip & EA & $\chi$ & $\eta$ & $\mathbf{S}$ & $\omega$ & $\log P$ & HE & Pol \\
\hline 1 & -9.2677 & -1.4469 & -9.8296 & 5.6382 & 4.1913 & .1193 & 3.7923 & 1.0200 & -2.8100 & 39.2100 \\
\hline 2 & -9.2766 & -1.4423 & -9.8462 & 5.6442 & 4.2020 & .1190 & 3.7908 & 1.0200 & -2.8100 & 39.2100 \\
\hline 3 & -9.2907 & -1.1641 & -9.8419 & 5.5030 & 4.3389 & .1152 & 3.4897 & 1.1900 & -2.4600 & 42.9700 \\
\hline 4 & -9.2361 & -1.3186 & -9.7722 & 5.5454 & 4.2268 & .1183 & 3.6376 & 2.6000 & -4.1600 & 52.5400 \\
\hline 5 & -9.1866 & -1.5947 & -9.6242 & 5.6094 & 4.0147 & .1245 & 3.9188 & 1.2400 & -3.9600 & 50.4600 \\
\hline 6 & -9.2919 & -1.1716 & -9.8395 & 5.5056 & 4.3340 & .1154 & 3.4969 & .8000 & -2.4400 & 41.1400 \\
\hline 7 & -9.3305 & -1.3926 & -9.8178 & 5.6052 & 4.2126 & .1187 & 3.7291 & .9700 & -2.8600 & 49.8200 \\
\hline 8 & -9.2096 & -1.1753 & -9.7423 & 5.4588 & 4.2835 & .1167 & 3.4784 & -.1900 & -2.9300 & 42.4000 \\
\hline 9 & -8.8345 & -1.6466 & -10.7411 & 6.1938 & 4.5472 & .1100 & 4.2183 & -.0600 & -7.2900 & 30.4100 \\
\hline
\end{tabular}

\section{Materials and Methods}

\subsection{Bioassay}

The super oxide dismutase assay i.e Super oxide radical scavenging activity has been carried out for nine chromone derivatives.

\subsection{Measurement of Superoxide Scavenging Activity}

The superoxide scavenging ability of the compound was assessed by the method of Winterbourn et al. (1975) [21 ]. Principle of this assay is based on ability of superoxide ion to inhibit the reduction of nitro-blue tetrazolium (NBT) to nitroblue tetrazolium formazon and is measured spectrophotometrically at $560 \mathrm{~nm}$. One unit is defined as that amount of enzyme causing half the maximum inhibition of NBT reduction. The reaction velocity will depend largely on somewhat variable assay conditions such as light intensity and reaction temperature.

\subsection{Reagents}

- 0.067 M Potassium phosphate buffer, pH 7.8

- $0.1 \mathrm{M}$ Ethylene diamine tetraacetic acid (EDTA) containing $0.3 \mathrm{mM}$ sodium cyanide
- $0.12 \mathrm{mM}$ Riboflavin (store cold in a dark bottle)

- $1.5 \mathrm{mM}$ Nitroblue tetrazolium (NBT) (store cold)

\subsection{Procedure}

Superoxide anions were generated in samples that contained in $3.0 \mathrm{ml}, 0.02 \mathrm{ml}$ of the chromone derivative $(10 \mathrm{mg})$, $0.2 \mathrm{ml}$ of EDTA, $0.1 \mathrm{ml}$ of NBT, $0.05 \mathrm{ml}$ of riboflavin and $2.64 \mathrm{ml}$ of phosphate buffer. The control tubes were also set up where DMSO was added instead of the chromone derivatives. All the tubes were vortexed and the initial optical density was measured at 560nm in a spectrophotometer (Genesys, 10-S, USA). The tubes were illuminated using a fluorescent lamp for 30 minutes (A foil-lined box approximately 4' long X 8" X 6" with an internally mounted $40 \mathrm{~W}$ fluorescent bulb has been used successfully). The absorbance was measured again at $560 \mathrm{~nm}$. The difference in absorbance before and after illumination was indicative of superoxide anion scavenging activity.

\section{Computational Calculations}

\subsection{Data Set}

The physicochemical parameters, such as vertical ionization potentials (IPv's) electron affinity (EA), electronega- 
tivity $(\chi)$, hardness $(\eta)$, softness $(S)$, electrophilic index $(\omega)$, partition coefficient $(\log \mathrm{P})$, charges, hydration energy $(\mathrm{HE})$ and polarisability $(\mathrm{Pol})$ were obtained forchromone derivatives.

\subsection{Molecular Structure Building}

A series of compounds tested for inhibitory activity was selected for the present study and the program of window Hyperchem software inc [22] (http://www.warezdestiny.com/free-hyp) was used in modeling studies. The molecules were generated and the energy was minimized using molecular modeling pro. The window version software SPSS10 [23] (SPSS Software. Consult http://www.spss.com )was used in the regression analysis.

\subsection{Validation of QSAR Models}

QSAR technique was applied to the analogs of chromone that were varied at the $\mathrm{R}$ and $\mathrm{R}^{1}$ position. The appropriate descriptors or parameters for the compounds, vertical ionization potentials $\left(\mathrm{IP}_{\mathrm{v}}\right.$ 's), electron affinity (EA), electronega$\operatorname{tivity}(\chi)$, hardness $(\eta)$, softness $(S)$, electrophilic index $(\omega)$, partition coefficient (Log $\mathrm{P}$ ) charges, polarisability $(\mathrm{Pol})$ and hydration energy (HE) were used as independent variables for deciding in poly [ADP-ribose] polymeraseinhibitory activity.

\subsection{Chemical Descriptors}

\subsubsection{Calculated Properties}

Quantum chemical calculations at the AM1[24,25] and PM3 [26] semiempirical theory levels, are employed for full optimization of the selected neutral compounds. The geometrical structures of the radicals studied are optimized independently from the neutral molecules prior to the calculation of energies, treated as open shell systems. All calculations are performed by using the program of window Hyperchem software Inc.

The calculated vertical ionization potential (IPv's) and electron affinity (EA) are corrected for zero-point energy, assuming a negligible error and thus saving computer-time. The IPv are calculated as the energy differences between a radical cation and the respective neutral molecule; $I P v\left(E_{c a-}\right.$ tion $\left.-\mathrm{E}_{\text {neutral }}\right)_{\mathrm{DFT}}$ and Koopmans's theorem ( $\mathrm{IPV}=-\varepsilon$ номо $)$. The EA are computed as the energy differences between a neutral form and the anion molecule; $\mathrm{EA}=\mathrm{E}_{\text {neutral }}-\mathrm{E}_{\text {anion }}$. The AM1 and PM3-based reactivity descriptors are obtained from Eqs. (1) - (4) [27-30].

\subsection{Correlation Analysis}

A relation between biological activity, expressed as Log1/mic, and the physicochemical parameters and QSAR was analyzed statistically by fitting the data to correlation equations consisting of various combinations of these parameters. The statistical optimization was used to propose the best correlation model.

The matrix correlation uses the Pearson product moment correlation to measure the degree of linear relationship be- tween two variables. The coefficient assumes a value between -1 and +1 .If one variable tends to increase the other decreases, the correlation coefficient is negative. Conversely, if the two variables tend to increase together the correlation coefficient is positive. We obtained the correlation matrix between inhibitory activity and respective calculated properties for 9 chromone derivatives. The more relevant regression models were selected for the following criteria: The correlation coefficient $(\mathrm{R})$, the Fisher ratio values $(\mathrm{F})$ and the standard deviations(s),standard error estimate (SEE), percentage of effective variable $(\% \mathrm{EV})$ and $\mathrm{R}^{2}$ adjusted( $\left.\mathrm{R}_{\text {adj }}^{2}\right)$.

The best equation was also tested for their predictive power using a cross- validation procedure .The crossvalidation is a practical and reliable method for testing this significance. In principle, the so-called "leave-one-out" approach consist in developing a number of models with one sample omitted at the time.

After developing each model, the omitted data is predicted and the differences between actual and predicted reduction potential $(\mathrm{y})$ values are calculated .The sum of squares of these differences is computed and finally the performance of the model (its predictive ability) is given by PRESS (Predictive Sum of Squares) and S SRESS (Standard deviation of cross validation ).

The predictive ability of the model was also quantified in terms of the $\mathrm{Q}^{2}[31]$.

\subsection{Docking Studies and Validation}

GOLD and Argus lab 4.0.1 is Molecular modeling and Drug Docking software's $[32,33]$.This helps in computational virtual screening to find the lead compounds. Molecular docking started with Fischer's lock and key theory, where, every receptor has its unique ligand to catalyze the reaction.

Argus Lab 4.0.1 is Molecular modeling and Drug Docking software. It is very flexible and can reproduce crystallographic binding orientations. Argus lab, which provides a user-friendly graphical interface and uses Shape Dock algorithm, was used to carry out docking studies of the poly [ADP-ribose] polymerase.

The Gold fitness was calculated by defining the site using the list of atom numbers and retaining all the other default parameters. Now a day's docking is frequently to predict the binding orientations of small molecules of drug candidates to their protein targets in order to predict the affinity of the small molecules [34].The 3D structure of poly [ADP-ribose] polymerase was retrieved from Protein Data Bank (PDB ID 3SE2) with an X-ray resolution of 2AO[35] http://www.rcsb.org/pdb). Docking poses were obtained by applying Goldscore, fitness functions available for scoring. As easily interpretable results were obtained based on a recently published work [36] all the results reported in the present paper are referred to the Chemscore fitness functions. These complexes were prepared for dockingstudies by addinghydrogen atoms, removing water molecules and co-crystallized inhibitors and refined by using 
the DeepView/SwissPdbViewer3.7(SP5)(Guex N, Peitsch MC. Swiss Model and the Swiss Pdb-Viewer: An environment for comparative protein modeling. Deep View/SwissPdbViewer3.7 (SP5) [37] Enzyme-inhibitor interactions within a radius equal to $15 \AA$ centered on reported bound inhibitors were taken into account. As a con- clusive part of docking we expect ,generated results should yield RMSD values below $1.5 \AA$. Successful docking has been performed for the selected set of 9 chromone inhibitors and their corresponding chemscore with their RMSD have been produced in the table 6 .

Table 6. Binding Energy, Chemscore and Gold fitness values of the docked ligands.

\begin{tabular}{|c|c|c|c|c|c|c|c|c|c|}
\hline comp & $\begin{array}{l}\text { Chem } \\
\text { Score }\end{array}$ & DG & S(hbond) & S(metal) & S(lipo) & DE(clash) & DE(int) & Gold fitness & $\begin{array}{l}\text { Argus bind- } \\
\text { ing energy }\end{array}$ \\
\hline 1 & 33.52 & -48.68 & 0.43 & 0.00 & 356.98 & 6.66 & 8.49 & -21.68 & -9.1987 \\
\hline 2 & 36.62 & -51.19 & 0.93 & 0.00 & 364.03 & 8.78 & 5.79 & -27.07 & -8.6971 \\
\hline 3 & 37.58 & -57.82 & 1.94 & 0.00 & 391.95 & 10.42 & 9.82 & 71.79 & -7.5616 \\
\hline 4 & 9.42 & -57.95 & 0.00 & 0.00 & 471.62 & 15.59 & 32.94 & -2025.27 & 0 \\
\hline 5 & -84.77 & -56.69 & 2.71 & 0.00 & 388.41 & 41.92 & 99.55 & -2687.50 & 0 \\
\hline 6 & 32.52 & -51.03 & 0.94 & 0.00 & 362.36 & 8.31 & 10.20 & -4.00 & -7.4987 \\
\hline 7 & -61.13 & -55.87 & 0.00 & 0.00 & 452.53 & 40.37 & 76.63 & -2563.42 & 0 \\
\hline 8 & -51.80 & -56.70 & 0.00 & 0.00 & 409.64 & 26.75 & 81.75 & -1655.79 & -7.9262 \\
\hline 9 & -16.87 & -42.52 & 0.92 & 0.00 & 323.36 & 0.10 & 59.29 & -1774.43 & -8.7536 \\
\hline
\end{tabular}

\section{Results and Discussions}

\subsection{Simple Linear Regression Model}

The biological activity data and the physicochemical propertiesIPv, IP, EA, EI, EN, Hard, Soft, LogP, HE and Pol of the chromone derivatives are given in Tables 1-3. The data from these tables were subjected to regression analysis. The Correlation matrices were generated with 9 analogs (supplementary material). The term close to 1 indicates high co-linearity, while the value below 0.5 indicates that no co-linearity exist between more than the two parameters.

The perusal of correlation matrix (supplementary material) indicates that Hardness is the predicted parameters from
AM1 method. The hardness $(\eta)$ gap between anti-bonding and bonding molecular orbitals, a reflection of the molecular stability [38]. From regression methods backward, forward, removed and stepwise.Hardness was found to be explainable variable. The regression technique was applied through the origin using these explainable parameters.

$$
\begin{aligned}
& \text { Activity =0.09571 (0.011) Hard } \\
& \mathrm{N}=9 ; \mathrm{R}=0.950 ; \\
& \mathrm{R} 2=0.903 ; \mathrm{R} 2 \mathrm{adj}=0.891 ; \\
& \% \mathrm{EV}=90.3 ; \\
& \mathrm{SEE}=0.1398 ; \\
& \mathrm{F}=74.642 ; \mathrm{Q}=6.7954
\end{aligned}
$$

In addition, the plot of observed activity versus predicted 
activity was not found to be satisfactory. Hence, the predictive ability of the model is not good.Eq. 1 shows that the values of $\% \mathrm{EV}$ are less and to improve its value, outliers were sought and eliminated.

After the elimination of the outlier (1, 5 and 8), a second model was developed. Overall, there is an increase in $\mathrm{R}$ and \%EV (90.3 - 97.4) values, and a decrease in SEE (0.1398-0.07368).

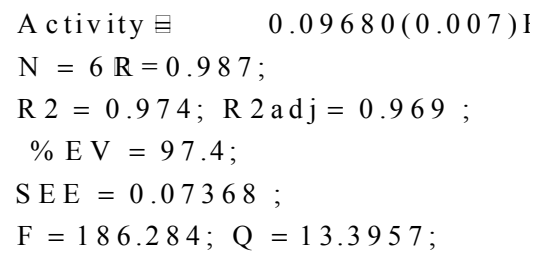

Eq. 2 is an improved model since it explains the biological activity to the extent of (97.4\%). In this way, the predictive molecular descriptors Hardness was considered as variable.

From the correlation matrix table, it reveals Hardness was found to be explainable variables. A uniparametric QSAR equation with Hardness was generated in PM3 method also.

$$
\begin{aligned}
& \text { Activity }=0.0945(0.011) \text { Hard } \\
& \mathrm{N}=9 ; R=0.951 ; \\
& \mathrm{R} 2=0.905 ; \mathrm{R} 2 \mathrm{adj}=0.893 ; \\
& \% \mathrm{EV}=90.5 ; \\
& \mathrm{SEE}=0.1385 ; \\
& \mathrm{F}=76.189 ; \mathrm{Q}=6.8664 ;
\end{aligned}
$$

Eq. 3 shows that the values of $\% \mathrm{EV}$ is less and to improve its value, outliers were sought and eliminated, In addition, the plot of observed activity versus predicted activity was not found to be satisfactory. Hence, the predictive ability of the model is not good. After the elimination of the outlier (1, 5 and 8), a second model was developed.

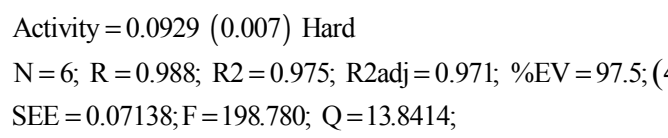

In an attempt to investigate the predictive potential of proposed models, the cross-validation parameters $(\mathrm{q} 2 \mathrm{cv}$ and PRESS) were calculated and used. The predictive power of the equations was confirmed by leave-one-out (LOO) cross-validation method [39] where, compounds are deleted one after another and prediction of the activity of the deleted compound is made based on QSAR model. The crossvalidation evaluates the validity of a model by how well it predicts the data rather than how well it fits the data. The cross-validation parameter, $\mathrm{q} 2 \mathrm{cv}$, is mentioned in the respective equations (Table 4 and 5).

\begin{tabular}{|c|c|c|c|c|c|}
\hline \multirow{2}{*}{\multicolumn{2}{|c|}{ compound observed }} & \multicolumn{2}{|l|}{ Eq(3) } & \multicolumn{2}{|l|}{ Eq.(4) } \\
\hline & & Predicted & Residual & Predicted & Residual \\
\hline 1 & .6778 & .3961 & 0.2817 & - & - \\
\hline 2 & .4686 & .3971 & 0.0715 & 0.3971 & 0.0715 \\
\hline 3 & .4949 & .4100 & 0.0849 & 0.4100 & 0.0849 \\
\hline 4 & .3373 & .3994 & -0.0621 & 0.3994 & -0.0621 \\
\hline 5 & .2677 & .3794 & -0.1117 & - & - \\
\hline 6 & .4437 & .4096 & 0.0341 & 0.4096 & 0.0341 \\
\hline 7 & .3099 & .3981 & -0.0882 & 0.3981 & -0.0882 \\
\hline 8 & .2147 & .4048 & -0.1901 & - & - \\
\hline 9 & .4090 & .4297 & -0.0207 & 0.4297 & -0.0207 \\
\hline
\end{tabular}

Table 4. Observed activity and predicted activity values of chromone derivatives by using AM1.

\begin{tabular}{llllll}
\hline & & Eq(3) & \multicolumn{3}{c}{ Eq.(4) } \\
compound & observed & Residual & Predicted & Residual \\
\hline $\mathbf{1}$ & & Predicted & Resin & - \\
$\mathbf{2}$ & .6778 & .3959 & 0.2817 & - & 0.0716 \\
$\mathbf{3}$ & .4686 & .3970 & 0.0716 & 0.3970 & 0.0922 \\
$\mathbf{5}$ & .4949 & .4027 & 0.0922 & 0.4027 & -0.0634 \\
$\mathbf{6}$ & .3373 & .4007 & -0.0634 & 0.4007 & - \\
$\mathbf{7}$ & .2677 & .3943 & -0.1266 & - & 0.0402 \\
$\mathbf{8}$ & .4437 & .4035 & 0.0402 & 0.4035 & -0.0856 \\
$\mathbf{9}$ & .3099 & .3955 & -0.0856 & 0.3955 & - \\
\hline
\end{tabular}

Table 5. Observed activity and predicted activity values of chromone derivatives by using PM3.

$$
q_{c v}^{2}=\frac{(S D-P R E S S)}{S D}
$$

where the PRESS (predictive residual sum ofsquares) and $\mathrm{SD}$ (standard deviation) valves are obtained as

$$
\begin{aligned}
\text { PRESS } & =\sum\left(\text { property }_{\text {observed }}-\text { property }_{\text {predicted }}\right)^{2}, \\
\mathrm{SD} & =\sum\left(\text { property }_{\text {observed }}-\text { property }_{\text {mean }}\right)^{2} .
\end{aligned}
$$

Eq. 3 and 5 of AM1 and PM3 methods respectively give a good $\mathrm{q}_{\mathrm{cv}}^{2}$ values, which should be always smaller than $\% \mathrm{EV}$. A model is considered to be significant when $\mathrm{q}_{\mathrm{cv}}^{2}>0.3$.

Another cross-validation parameter, PRESS which is the sum of the squared differences between the actual and that 
predicted when the compound is omitted from the fitting process, also supports the predictive ability of Eqs.2 and 4. Its value decreases from Eq. 1 to Eq.3.

The quality factor $\mathrm{Q}$, is defined as the ratio of regression constants (R)to the standard error estimation (SEE), that is , $\mathrm{Q}=\mathrm{R} / \mathrm{SEE}$. This indicates that the higher the value of $\mathrm{R}$, and the lower the value of SEE, the higher is the magnitude of $\mathrm{Q}$ and the better will be the correlation. In present case, Q increases from 6.7954 to 13.3957 and 6.8664 to 13.8414 (Eq. 1 to 4 ).

\subsection{Docking Analysis}

The compounds were docked using each of the two docking software's. The Chemscore, GOLD Score and Argus binding energy values are given in Table 6 . The binding energies obtained in Argus Lab ranged from -7.9262to $9.1987 \mathrm{~kJ} / \mathrm{mol}$.The results of Gold are analyzed in terms of Chem score (Chem score ranging from 37.58 to -84.77) and Gold fitness(71.79 to -2687.50 )(Table 6).

Docking results revealed that electron withdrawing substituent group (R1) on the compounds play a key role in ligand binding interactions with protein. The predicting scoring functions of these compounds have shown good correlation with Argus binding energy values.

Molecule 3 has best Chem score(37.58) andGold fitness(71.79) with good binding energy $(-7.5616 \mathrm{Kcal} / \mathrm{mol})$ compared to all other molecules and has best vander waal interactions and Hydrogen bonding interactions with optimum clash penalty $(\mathrm{DE}$ (clash) $=10.42)$ and showing best lipophilic character $(\mathrm{S}($ lipo $)=391.95)($ Table 6$)$. All the poses of molecule 3(chosen as best in docking studies) and its interactions in the active pocket of poly [ADP-ribose] polymerase have been illustrated in figure5.

The docking simulation of the most active chromone derivatives 9 toward poly [ADP-ribose] polymerase (PDB ID 3SE2) showed that the most enzyme-inhibitor complex was stabilized by hydrophobic interactions occurring between the aromatic moieties of the ligand and lipophilic residues of the binding site. In particular the compound 3 group was oriented towards the hydrophobic region lined by Ile857, Arg726, His523, His518, His513 and Ser510.

\section{Conclusion}

In conclusion, the model developed to predict the structural features of chromone derivatives to inhibit; poly [ADP-ribose] polymerase reveals useful information about the structural requirement for the molecule. The present study showed that the QSAR studies and the docking approach of chromone derivatives as poly [ADP-ribose] polymerase inhibitors are, successfully modeled using mono parametric equations. The best predictive AM1 model resulted in cross-validated $\mathrm{R}^{2}$ value of $0.974, \mathrm{R}^{2}$ adj value of 0.969 and standard error of estimate 0.07368 (AM1). Similarly the best predictive PM3 model was derived with $\mathrm{R}^{2}$ of $0.975, \mathrm{R}^{2}$ adj of 0.971 and standard error of estimate of 0.07138 , comprising hardness. These models were able to predict the activity of test set molecules efficiently (best molecules,3 ) within an acceptable error range. GOLD and Argus lab 4.0.1 were employed to dock the inhibitors into the active site of poly [ADP-ribose] polymerase and these docking studies revealed the vital interactions viz. hydrogen bonding interactions, vanderwaal interactions and binding conformation of the inhibitors(Fig-4).

The Eq. 2 and 4 from AM1 and PM3, semi empirical calculations reveal Hardness cause the inhibitory activity.Higher values of Hardness is responsible for higher inhibitory activity nature for poly [ADP-ribose] polymerase enzyme. Hardness $(\eta=1 / 2($ HOMO_LUMO $))$ represents a variable, statistically related to antioxidant activity. According to Pearsons HSAB principle hard applies to species which are small, have high charge states and weakly polarisable .The maximum hardness and minimum polarizability criterion complement the minimum energy criterion for stability. In general, a stable state or a favourable process is associated with maximum hardness and minimum polarizability[40].Therefore hardness values are favourable for higher inhibitory activity. The linear dependence of inhibitory nature on Hardness was evident from Figure 3 and 4.

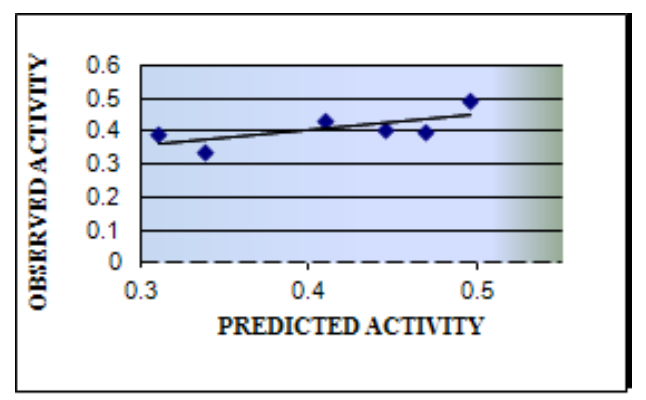

Fig. 3. Plot of Observed Verses Predicted activity (AM1 Method).

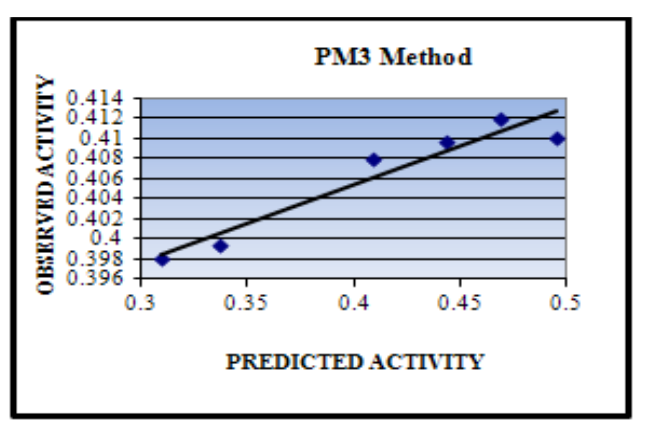

Fig. 4. Plot of Observed Verses Predicted activity (PM3 Method).

The most active compounds were docked successfully into the active site of the inhibited enzyme. Inhibitory activity of the most potent compounds was explained mostly by hydrogen bonding interactions, vander waals interactions and lipophilic character. Hydrogen bonds, like any other bonds, provide binding energy to the protein-ligand complex. The significance of a particular hydrogen bond to a particular protein-ligand complex is dependent on the geometry and distance of the bond, partial charges on the donor or acceptor. Lipophilic (Hydrophobic) interactions play a key role in the folding and maintenance of the 3- 
dimensional structure of proteins, as well as in the binding of ligands (e.g. drugs) to protein targets. Molecules can attract each other at moderate distances and repel each other at close range. These forces are collectively called "van der Waals forces".

The compound 3 was found to present high antioxidant activiy,and siginificant inhibitory activity on poly [ADPribose] polymerase. High antioxidant activity of compound 3 compared to other compounds is due to more hydrogen bonding interactions, vander waals interactions, lipophilic character and more electronegative or more electron withdrawing substituent group (R1) on the compound (Table 1 and 8). The QSAR equations suggested that electronega- tive group on benzoyl ring play an important role for antioxidant activity [41]. These electronegative substituents might help in the radical stabilization throughout the chromone nucleus. The steric descriptors indicated that the bulky substituents near position $\mathrm{R}$ in chromone (Fig-2) were disfavored. Steric hindrance around these regions may interfere with the planarity between ring and carbonyl group of the chromone nucleus, therefore affecting radical delocalization. The best protein-ligand interactive poses of molecules 3 (figures 5) show good hydrogen bonding energies. The more effective and the higher activity of the chromone compound - 3 (Figure 5).

Table 1. Inhibition effect of Chromone derivatives by Super Oxide Scavanging activity method.

\begin{tabular}{|c|c|c|c|c|c|}
\hline Comp & derivatives & $\mathbf{R}$ & $\mathbf{R}^{\prime}$ & $\operatorname{MIC}(\mu \mathrm{g} / \mathrm{ml})$ & $\begin{array}{l}\text { Activity } \\
\text { MIC=Log1/MIC }\end{array}$ \\
\hline 1 & chromone & & & 21 & -1.3222 \\
\hline 2 & chromone & & & 34 & -1.5314 \\
\hline 3 & chromone & & & 32 & -1.5051 \\
\hline 4 & chromone & & & 46 & -1.6627 \\
\hline 5 & chromone & & & 54 & -1.7323 \\
\hline 6 & chromone & & & 36 & -1.5563 \\
\hline 7 & chromone & & & 49 & -1.6901 \\
\hline 8 & chromone & & & 61 & -1.7853 \\
\hline 9 & chromone & & & 39 & -1.5910 \\
\hline
\end{tabular}<smiles>[R]c1oc2c3c(ccc2c(=O)c1C)OC([R])CC3=O</smiles>

Figure 2. Structural skeleton Chromone derivatives.

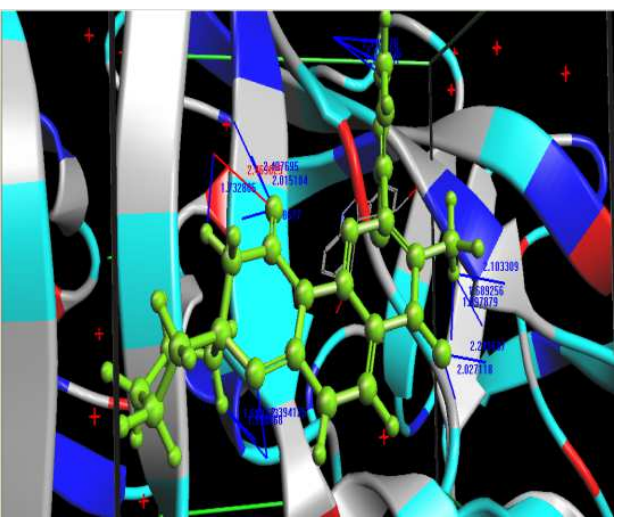

Figure 5. Binding orientation of molecule-3 in poly [ADP-ribose] polymerase(PDB ID 3SE2). 
Binding orientation of green coloured Ballcylinder low model molecule 3 in Argus dock and crystallographic conformation of active site of poly [ADP-ribose] polymerase(PDB ID 3SE2).Hydrogen bonds are shown in red colour dotted lines.

\section{Acknowledgements}

Authors are thankful to the Head department of chemistry, University College of Science, Saifabad, Osmania University, for providing the facilities for the research work and Muffakhamjah College of Engineering and Technology, Hyderabad.

\section{References}

[1] Miao H, Yang Z (2000) Regio-specific carbonylative annulation of iodophenol acetates and acetylenes to construct the flavones by a new catalyst of palladiu-thiourea-dppp complex. Org Lett 2: 1765 and refs. therein.

[2] Silva AMS, Pinto DCGA, Cavaleiro JAS, Levai A, Patonay $\mathrm{T}$ (2004) Synthesis and reactivity of styrylchromones. Arkivoc 106 (c) Levai A (2004) Synthesis of exocyclic $\alpha, \beta-$ unsaturated ketones. Arkivoc 15.

[3] Cutting WC, Dreisbach R H, Azima M,NeffBJ, Brown BJ, Wray J (1951) Antiviral chemotherapy. V. Further report on flavonoids. Stanford Med Bull 9:236.

[4] Mentzer C, MeunierP, Lecocq J, Billet D, Xuong D (1945) Bull Soc Chim Fr 12:430 (c) Cox JSG (1967) Nature, London 216: 1328 (d) Orr TSC, Pollard MC, Gwilliam J (1970) Cox JSG Mode of action of disodium Cromoglycate, studies on immediate type hypersensitivity reactions using 'double sensitization' with two antigenetically distinct rat regains. Celin Exp Immunol 7: 745.

[5] Valenti P, Bisi A, Rampa A, Belluti F, Gobbi S, Zampiron A, Carrara M(2000) Synthesis andbiological activity of some rigid analogues of flavone-8-acetic acid.Biorg Med Chem 239.

[6] Lim LC, Kuo YC, Chou CJ (2000) Cytotoxic biflavonoids from Selaginelladelicatula. J Nat Prod63:627.

[7] Shi YQ, Fukai T, Sakagami H, Chang WJ, Yang PQ, Wang FP, Nomura T (2001) Cytotoxic flavonoids with isoprenoid groups from Morusmongolica. J Nat Prod 64:181.

[8] Larget R, Lockhart B, Renard P, Largeron M (2000) A Convenient Extension of the Wessely Moser Rearrangement for the Synthesis of Substituted Alkyl amino flavones as Neuroprotective Agents In Vitro Biorg Med Chem Lett 10: 835.

[9] Groweiss A, Cardellins JH, Boyd MR (2000) HIVInhibitory prenylatedxanthones and flavones from Macluratinctoria. J Nat Prod 63:1537.

[10] Deng Y, Lee JP, Ramamonjy MT, Synder JK, Des Etages SA, Kanada D, Synder MP,Turner CJ(2000) New antimicrobial flavanones from Physenamadagascariensis. J Nat Prod 63:1082.

[11] Khan IA, Avery MA,Burandt CL, Goins DK, Mikell JR, Nash TE, Azadega A,Walker LA (2000) Antigiardial activity of isoflavones from Dalbergiafrutescens bark. J Nat Prod 63: 1414.

[12] .Mori K, Audran G, Monti H (1998) ChemInform Abstract: The First Synthesis of Coniochaetones A and (.+-.)-B: Two Benzopyranone Derivatives. Synlett 259.

[13] Pietta PJ (2000) Flavonoids as antioxidants. J Nat Prod 63:1035.

[14] (a)Beecher GR (2003) Overview of Dietary Flavonoids: Nomenclature, Occurrence and Intake J Nutr 133:3248S. (b) Hoult JRS, Moroney MA, Paya M (1994) Actions of flavonoids and coumarinson lipoxygenase and cyclooxygenase. Methods Enzymol 234:443.

[15] Horton DA, Bourne GT, Smythe ML (2003) The combinatorial synthesis of bicyclic privileged structures or privileged substructures. ChemRev 103: 893.

[16] Grindlay D, Reynolds T (1986) The 2008 aloe vera phenomenon. J Ethnopharmacology16:117.

[17] Hirata T, Suga T (1978) Biosynthesis of Aloenin in Aloe arborescence var.natalensis. Bull Chem Soc Jap 51:842.

[18] Womble D, Helderman JH (1988) Enhancement of alloresponsiveness of human lymphocytes by acemannan(carrisyn).Int J Immunopharmac 10: 967.

[19] Yu D, Brossi A, Kilgore N, Wild C, Alloway G, Lee KH. (2003) Anti-HIV agents. Part 55: 3'R, 4'R- Di-(O)-(-)camphanoyl-2', 2'-dimethyldihydropyrano [2, 3-f] chromone (DCP), a novel anti-HIV agent. Bioorg Med Chem Lett 13(9):1575.

[20] (a) Bouanani H, Gayoso JCR (1973). Acad Sci Paris Ser C 273:399 (b) Saburi Y, Yoshimoto T, Minami K (1971) Nippon Kagaku Zaisshi 92: 552.

[21] Dias MM, Machado NFL, Marques MPM (2011) Dietary Chromones as antioxidant agents-The Structural variable. Food and function 2:595.

[22] Pal pacher, Csalba S, (2008) Role of the peroxynitrile-PARP pathwayin human disease. The American Journal of Pathology 1(173):2-13.

[23] Winterbourn CC, Hawkins RE, Brian M, Carrell RW(1975) The estimation of red cell superoxideDismutase activity. J Lab Clin Med. Feb 85(2):337-41.

[24] http://www.warezdestiny.com/free-hyp.

[25] SPSS Software. Consult http://www.spss.com.

[26] Roothan CCJ (1951) New Developments in Molecular Orbital Theory. Rev Mod Phys 23:69.

[27] Pople JA, Nesbet RK (1954) Self consistent Orbitals for Radicals. J Chem Phys 22:571.

[28] McWeeny R, Dierksen G (1968) Interpolating functionals in relation to the transition state and transition operator methods. J Chem Phys 49: 4852.

[29] Dewar MJS, Zoebisch EG, Healy EF, Stewart JJP (1985) The development and use of quantum mechanical molecular models 76. AMI: a new general purpose quantum mechanical molecular mode. J Am Chem Soc 107:3902.

[30] Stewart JJP (1989) Optimization of parameters for semi 
empirical methods. J Comput Chem 10:209.

[31] Kohn W, Becke AD, Parr RG Density Functional Theory of Electronic Structure. J Phys Chem10:12974.

[32] Parr RG, Pearson RG (1983) Hardness, softness, and the Fukui function in the electronic theory of metals and catalysis. J Am Chem Soc 105:7512.

[33] .Rameshwar N, Krishna K, Ashok Kumar B, Parthasarathy T (2006) QSAR studies of N1-(5-chloro-2- pyridyl)-2- $\{[4-$ (alkyl methyl)] amino -5 -chlorobenzamide analoges. Bio org Med Chem 14:319-325.

[34] Jones G, Willet P, Glen RC (1995) Molecular recognition of receptor sites using a genetic algorithm with a description of desolvation. J mol bio 245:43.

[35] Thompson \& Mark A. ArgusLab 4.0.1 www.arguslab.com Planaria Software LLC, Seattle WA.

[36] Pogliani L (1994) Structure property relationships of amino acids and some dipeptides. AminoAcids. 6:14.

[37] Schulz-Gasch T, Stahl M (2004) Scoring functions for protein-ligand interactions": a critical perspective.Drug Discov
Today. 1 (3):231-239.

[38] Siemoneit U, Hofmann B, Kather L, Lamkemeyer T, Madlung J, Franke L, Schneider G, Jauch J, Poeckel D, Werz O (2008) Identification and functional analysis of cyclooxygenase-1 as a molecular target of boswellic acids. oche .Pharmacol. 71, $503-513$.

[39] Guex N, Peitsch MC. Swiss Model and the Swiss PdbViewer: An environment for comparative protein modeling. Deep View/SwissPdbViewer3.7 (SP5) Electrophoresis. 18: 2714-2723.

[40] Gilmann JJ (1997) Chemical and physical Hardness. Mater Res Innov 1:71-76.

[41] Chattterjee S, Hadi AS, Price B (2000) Regression Analysis by Examples 3rd Ed Willy: New York.

[42] Parr RG, Chattaraj PK (1991) Principle of maximum Hardness. J Am Chem Soc 113:1854.

[43] Weerasak S, Patcharawee N, Jiraporn U(2008)3D-QSAR Investigation of Synthetic Antioxidant Chromone Derivatives by Molecular Field Analysis Int J Mol Sci March 9(3): 235-246. 